Case Report

\title{
Acute Aortic Dissection with Intestinal Ischemia: What to Do First
}

\author{
Francesco Meriggi, MD, ${ }^{1}$ Alessia Alloni, MD, ${ }^{2}$ Paolo Gramigna, MD, ${ }^{1}$
}

Paola Tramelli, MD, ${ }^{1}$ and Mario Vigano, $\mathrm{MD}^{2}$

\begin{abstract}
Purpose: In the case of an acute aortic dissection, a surgical aortic procedure is usually the priority in order to restore the perfusion of vital organs. Afterwards, associated ischemic abdominal visceral lesions can be resected. For particular patients, it could be highly beneficial to perform the abdominal surgery before surgically addressing the aorta. The aim of this paper is to contribute to the therapeutic choice in cases of acute aortic dissection with acute abdomen.

Case report: The case is reported of a 38-year-old patient, affected by an acute aortic dissection (Stanford type A) and peritonitis.

Suspecting the necessity for a complex combined surgical procedure, the patient underwent emergency diagnostic laparoscopy, which showed an infarctual necrosis of the distal ileum and right colon. Therefore, he immediately underwent a wide right hemicolectomy. Afterwards, an ascending aortic substitution was performed.

The patient was discharged on the 15th post-operative day, and he is doing well, 1 year and 3 months after the operation.

Conclusion: In the case of an acute aortic dissection with acute abdomen, emergency laparoscopy is a precious surgical technique to identify criteria that can lead to therapeutic decisions, including timing.
\end{abstract}

Keywords: aortic dissection, intestinal malperfusion, laparoscopy

\section{Introduction}

Acute aortic dissection is one of the most frequent causes of vascular acute abdomen. ${ }^{1,2)}$ This pathology can cause serious complications, such as aortic rupture with consequent immediate death, visceral ischemic necrosis (bowel, liver, kidney) with possible mortal effects and

${ }^{1}$ Department of Surgical Sciences, University of Pavia, IRCCS San Matteo Hospital Foundation, Pavia, Italy

${ }^{2}$ Cardiac Surgery Division, University of Pavia, IRCCS San

Matteo Hospital Foundation, Pavia, Italy

Received: November 25, 2010; Accepted: February 7, 2011 Corresponding author: Francesco Meriggi, MD. Viale Liberta 35, 27100 Pavia, Italy

Email: f.meriggi@smatteo.pv.it

(C)2011 The Editorial Committee of Annals of Thoracic and Cardiovascular Surgery. All rights reserved. ischemia of the spinal cord leading to paraplegia. ${ }^{3)}$

Early diagnosis and surgical treatment can modify patient survival. ${ }^{4)}$

Aortic dissections with a coexisting impairment of visceral perfusion require combined surgical therapy. ${ }^{5)}$

One main decision is whether to choose a combined surgical approach: aortic repair and abdominal intervention at the same time, or to address pathologies separately at different times, after deciding which pathology must be addressed first. The choice is highly important. ${ }^{6}$ )

Nowadays, emergency laparoscopy ${ }^{7,8)}$ can contribute to resolve significant diagnostic and differential diagnostic questions in the presence of acute abdominal symptomatology. It offers the advantage of being feasible at the bedside (in the Intensive Care Unit or Emergency room) of critical patients who cannot be moved, avoiding useless and risky exploratory laparotomies. 


\section{Case Report}

The case is reported of a male, 38 years old, obese, a smoker, and affected by hypertension.

On July 13th 2009, he was hospitalized in his town for retrosternal pain extended to the abdomen and left lower limb. The next day he was operated on, in order to revascularize his limb by a right-left femoro-femoral by-pass for acute ischemia.

Because of the persistent pain, a CT scan was performed leading to the diagnosis of an acute aortic dissection (Stanford type A) ${ }^{2,3)}$ and showing a partially thrombosed false channel, extended from the ascending aorta to the left iliac bifurcation. The site of entry was in the right-anterior wall of the ascending aorta, two centimetres above the aortic commissures. Also the superior mesenteric artery, originating from the thrombosed false channel, could not be visualized distally from its origin, whereas the intimal flap extended into the coeliac artery indicating compression of the true channel (Fig. 1).

On July 15th 2009, the patient was referred to the Division of Cardiac Surgery at San Matteo Hospital of Pavia. At the moment of admission the patient had abdominal tenderness with bowel distension and mucous diarrhea. Due to visceral ischemia he was peritonitis with a septicaemic shock moderately hypotensive (blood pressure $=$ 95/50 $\mathrm{mmHg}$; pulse rate $=130$ beats $/ \mathrm{min}$ ); however, a transthoracic echocardiography excluded a cardiac tamponade. Also, the jugular central venous pressure was not elevated $(\mathrm{CVP}=4 \mathrm{mmHg})$. A profound metabolic acidosis was found (arterial blood $\mathrm{pH}=7.3$; serum $\mathrm{HCO}_{3^{-}}=6 \mathrm{mEq} / \mathrm{L}$; arterial lactate $=16 \mathrm{mmol} / \mathrm{L})$. White cell count was $25000 / \mathrm{mm}^{3}$ and serum creatinine level $1.5 \mathrm{mg} / \mathrm{dL}$.

The clinical picture of peritonitis and septic shock induced us to transfer the patient to the operating room and perform, on the table, an emergency laparoscopy to confirm the diagnosis; it showed an infarction of distal ileum and right colon with a serum-hematic effusion.

He immediately underwent, by way of median xifopubic laparotomy, a wide right hemicolectomy extending into the distal ileum with a latero-lateral ileum-transversal colon anastomosis. The patient remained stable during the procedure. After the bowel resection, the arterial pressure remarkably increased in association with a reduction of lactic acidosis (arterial lactate $=9.5 \mathrm{mmol} / \mathrm{L}$ ).

After the laparotomy on the same day, a median sternotomy was performed, along with a substitution of the ascending aorta, with a $28 \mathrm{~mm}$ Hemashield tubular prosthesis. Femoral and right subclavian artery and femoral

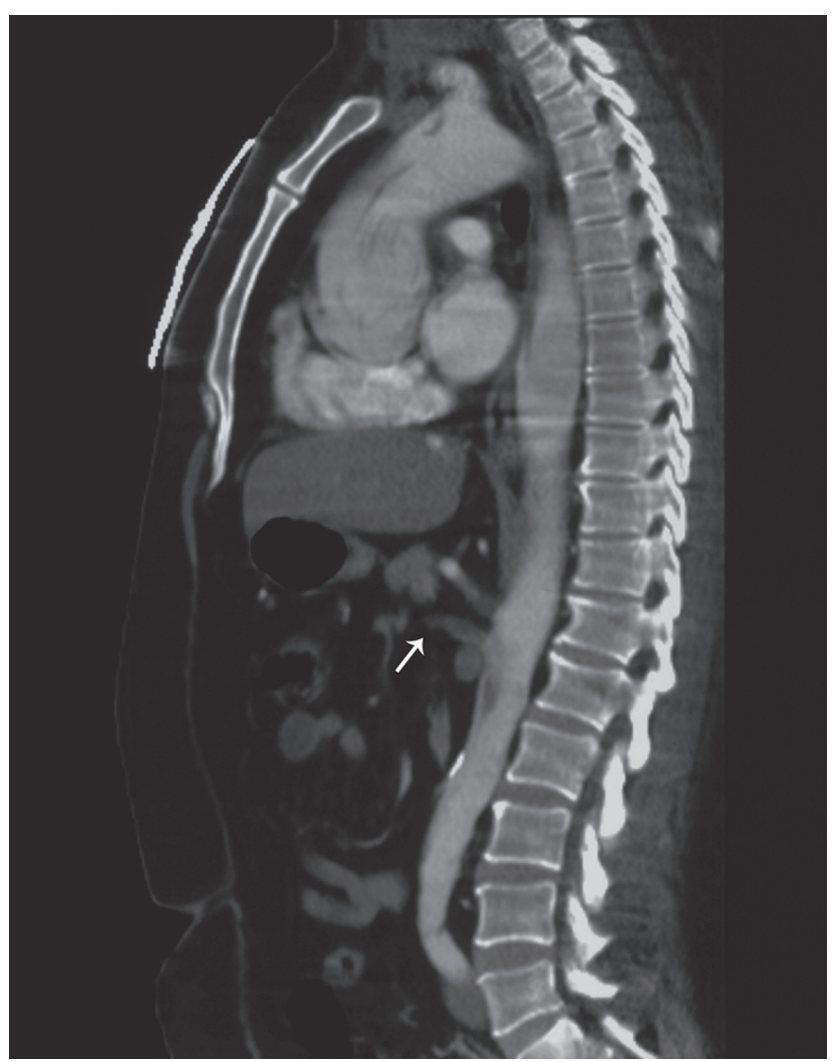

Fig. 1 CT scan. Acute aortic dissection (Stanford type A). The superior mesenteric artery can not be visualized distally from its origin (arrow).

veins were cannulated, and a 6 minute hypothermic $\left(20^{\circ} \mathrm{C}\right)$ circulation arrest was performed with a 35 -minute selective carotid cerebral perfusion. The distal anastomosis was performed with an open technique without clamping. At the end of cardiopulmonary by-pass, an electrocardiography showed right ischemia, which demonstrated the need for a right coronary by-pass. During surgery, EEG was continuously monitored.

The post-operative course was complicated by atrial fibrillation, which regressed after digitalic therapy. There were no other apparent complications.

The histological examination showed transmural necrosis of the small and large intestine.

The patient was discharged on the 15th post-operative day, and he is doing well, 1 year and 3 months after the operation. Actually, he refused any further radiological investigation.

\section{Discussion}

Visceral ischemia is a rare but severe complication of an aortic dissection, usually due to obliteration of 
splanchnic arteries by the intimal flap. The post-operative mortality can reach $80 \%$, when laparotomic and vascular procedures are combined.

Early diagnosis and surgical treatment can reverse ischemia. ${ }^{3)}$

Surgical therapy requires hypothermia and selective cerebral perfusion without clamping. Hypothermia itself can cause coagulation alterations and profuse bleeding, especially in patients with long cardiopulmonary by-pass and pre-existent coagulation diseases.

Nevertheless hypothermia can preserve internal organs from ischemic damage. Femoral and subclavian cannulation assure an adequate organ perfusion. Cerebral perfusion, with low temperature, steroids and barbiturates, preserves cerebral integrity. An open technique without clamping can also reduce the risk of embolism.

With reference to our case report, we underwent the patient an unusual surgical approach because of the not appropriate diagnostic management during his first hospitalization.

In fact, most surgeons perform the aortic surgery first because of the high risk of aortic rupture, followed by visceral and peripheral surgery. ${ }^{3,4)}$ Moreover, the aortic repair usually beneficially restores visceral perfusion with a possible regression of the symptoms related to the recent ischemia.

In few cases, ${ }^{9}$ if the aortic pathology is relatively stable in satisfying hemodynamic conditions and the hypertension is well controlled, and if the visceral ischemia is severe and considerable, it could be very important to perform the abdominal surgery before surgically addressing the aorta, in order to avoid further irreversible ischemic damages due to the circulation arrest in organs with compromised perfusion. ${ }^{6)}$ This was the case of our patient. Due to obesity, peritonitis and poor bowel preparation, we preferred an open approach for intestinal exeresis to minimize the risk of post-operative complications. ${ }^{7}$ 10)

In conclusion, considering the treatment of an acute aortic dissection complicated by a vascular acute abdomen, any attempt of abdominal visceral revascularization through fenestration, bypass surgery or primary repair should be challenged against the need of primary visceral resection. ${ }^{4,5)}$ In this perspective, emergency laparoscopy is a precious surgical technique to identify the criteria, that can lead to therapeutic decisions, including timing. These criteria should be an assessment of 1) site of visceral ischemia, 2) extension and degree of ischemia as well as 3) anticipation about the outcome of the failing organ (irreversibility). Clinical status, together with the identification of bowel ischemia, will facilitate the surgical decision.

\section{References}

1) Miani S, Boneschi M, La Penna A, et al. Acute vascular abdomen. General outlook and algorithms. Minerva Cardioangiol 1999; 47: 285-300.

2) Schmitto JD, Popov AF, Coskun KO, et al. Morphological investigations of type A aortic dissection. Ann Thorac Cardiovasc Surg 2010; 16: 331-4.

3) Khan IA, Nair CK. Clinical, diagnostic, and management perspectives of aortic dissection. Chest 2002; 122: $311-28$.

4) Fabre O, Vincentelli A, Willoteaux S, et al. Preoperative fenestration for type A acute aortic dissection with mesenteric malperfusion. Ann Thorac Surg 2002; 73: 950-1.

5) Okita Y, Takamoto S, Ando M, et al. Surgical strategies in managing organ malperfusion as a complication of aortic dissection. Eur J Cardiothorac Surg 1995; 9: 242-6; discussion 247.

6) Chang RW, Chang JB, Longo WE. Update in management of mesenteric ischemia. World J Gastroenterol 2006; 12: 3243-7.

7) Warren O, Kinross J, Paraskeva P, Darzi A. Emergency laparoscopy: current best practice. World J Emerg Surg 2006; 1: 24-32.

8) Zamir G, Reissman P. Diagnostic laparoscopy in mesenteric ischemia. Surg Endosc 1998; 12: 390-3.

9) Firstenberg MS, Sai-Sudhakar CB, Sirak JH, et al. Intestinal ischemia complicating ascending aortic dissection: first things first. Ann Thorac Surg 2007; 84: e8-9.

10) Pikarsky AJ, Saida Y, Yamaguchi T, et al. Is obesity a high-risk factor for laparoscopic colorectal surgery? Surg Endosc 2002; 16: 855-8. 